\title{
Three-Dimensional Vector Holograms in Photoreactive Anisotropic Media
}

\author{
Tomoyuki Sasaki ${ }^{1}$, Akira Emoto ${ }^{2}$, Kenta Miura ${ }^{1}$, \\ Osamu Hanaizumi ${ }^{1}$, Nobuhiro Kawatsuki ${ }^{3}$ and Hiroshi Ono ${ }^{2}$ \\ ${ }^{1}$ Department of Electronic Engineering, \\ Graduate School of Engineering, Gunma University, \\ ${ }^{2}$ Department of Electric Engineering, Nagaoka University of Technology, \\ ${ }^{3}$ Department of Materials Science and Chemistry, Graduate School of Engineering, \\ University of Hyogo, \\ Japan
}

\section{Introduction}

Polarization gratings, in which optical anisotropy is periodically modulated, are very attractive from the point of view of their interesting optical properties, including polarization selectivity of the diffraction efficiency and polarization conversion in the diffraction process (Nikolova et al., 1984). These properties make polarization gratings useful for numerous optical applications related to polarization discrimination (Davis et al., 2001; Asatryan et al., 2004), control (Nikolova et al., 1997; Hasman et al., 2002), and measurement (Gori, 1999; Provenzano et al., 2006). Polarization gratings can be fabricated by holographic exposure with polarized interference light in photoanisotropic media such as azobenzene-containing polymer (azopolymer) films (Todorov et al., 1984; Ebralidze et al., 1992; Huang and Wagner, 1993; Samui, 2008). Since azobenzene molecules reorient in accordance with polarization of light resulting from trans-cis-trans photoisomerization reactions, periodic structures are formed as spatial distribution of the molecular orientation by holographic recording using vectrial light (i.e., by vector holography). In addition, surface relief gratings are obtained by holographic recording in azopolymers (Rochon et al., 1995; Kim et al., 1995; Ramanujam et al., 1996; Naydenova et al., 1998; Labarthet et al., 1998, 1999). Nikolova et al. have investigated the diffraction properties of various types of vector holograms recorded in side-chain azobenzene polyesters (Nikolova et al., 1996). Birabassov and Galstian have analyzed the relationship of polarization states between holographic recording and reconstruction beams with the use of azopolymers (Birabassov et al., 2001). It has been also demonstrated that vector holograms realize recording and reconstruction of polarized optical images (Ono et al., 2009a, 2009b).

In a previous paper, we presented three-dimensional (3D) vector holography, which is a novel concept for holographic recording using vectrial light (Sasaki et al., 2008). Common vector holograms are recorded in initially isotropic media such as amorphous azopolymers. In contrast, 3D vector holograms are recoded in initially anisotropic media. Since the propagation velocity of light in anisotropic media varies in accordance with the polarization state, standing waves with a multidimensionally varying state of polarization were obtained in the region of overlap. We recorded anisotropic gratings in a liquid crystalline medium by 
the 3D vector holography, and investigated their diffraction properties. As a result, it was demonstrated that the $3 \mathrm{D}$ vector holography enables formation of higher-order periodical modulation of optical anisotropy. In addition, we observed that the multidimensional distribution exhibited interesting diffraction properties.

The present chapter reported the principle of 3D vector holography in detail, and investigated optical characteristics of 3D vector holograms recorded in a photoreactive anisotropic medium. Interference of two polarized light in anisotropic media was described by employing the extended Jones matrix method. Diffraction properties of the holograms, recorded in the model medium, were observed and the obtained results were analyzed through the use of the finite-difference time-domain (FDTD) method. By comparing the experimental and calculated results, we discussed the adequacy of the formation mechanism of the 3D vector holograms. In addition, the diffraction efficiency was simulated for various recording conditions to reveal the relationship between the multidimensional structure and the optical behaviour.

\section{Principle of three-dimensional vector holography}

We consider interference of mutually coherent polarized light in uniaxial anisotropic media. As illustrated in Fig. 1, the $x z$-plane is the incident plane and the $z$-axis is taken normal to the film plane. Assuming that the two recording beams are plane waves and that the amplitude of their incident angles is small, the electric field of interference light is described using the Jones vector as

$$
\mathbf{E}(x, z)=\mathbf{J}_{1}(z) \mathbf{E}_{1} \exp \left(i k_{1} x\right)+\mathbf{J}_{2}(z) \mathbf{E}_{2} \exp \left(i k_{2} x\right),
$$

where $\mathbf{J}_{1}$ and $\mathbf{J}_{2}$ are the extended Jones matrixes of the medium for the two beams, $\mathbf{E}_{1}$ and $\mathbf{E}_{2}$ are the Jones vectors of the two beams, $k_{1}=(2 \pi / \lambda) \sin \theta_{1} \equiv k \sin \theta_{1}, k_{2}=k \sin \theta_{2}, \theta_{1}$ and $\theta_{2}$ are the incident angles of the two beams, and $\lambda$ is the wavelength in a vacuum. Here, to simplify the analysis, reflection at the air-medium boundaries is neglected. When the ordinary and extraordinary indexes and the direction of the optical axis $\mathbf{c}_{0}$ are homogeneous in the medium, the extended Jones matrix is given by (Lien, 1990, 1997)

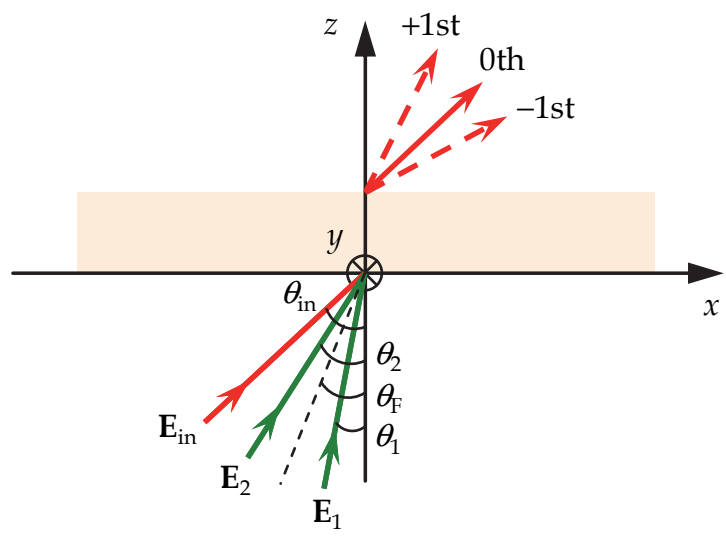

Fig. 1. Schematic illustration of arrangement for holographic recording and reconstruction. $\mathbf{E}_{\text {in }}$ is the electric field vector of reconstruction light 


$$
\mathbf{J}_{m}=\mathbf{S}_{m} \mathbf{G}_{m} \mathbf{S}_{m}^{-1}
$$

where

$$
\begin{aligned}
& \mathbf{G}_{m}=\left[\begin{array}{cc}
\exp \left(i k_{z 1} z\right) & 0 \\
0 & \exp \left(i k_{z 2} z\right)
\end{array}\right],(0 \leq z \leq d), \\
& \mathbf{S}_{m}=\left[\begin{array}{cc}
1 & c_{2} \\
c_{1} & 1
\end{array}\right], \\
& k_{z 1} / k=\sqrt{n_{\mathrm{o}}^{2}-\left(k_{m} / k\right)^{2}}, \\
& \frac{k_{z 2}}{k}=-\frac{\varepsilon_{x z}}{\varepsilon_{z z}} \frac{k_{m}}{k}+\frac{n_{\mathrm{o}} n_{\mathrm{e}}}{\varepsilon_{z z}} \sqrt{\varepsilon_{z z}-\left(1-\frac{n_{\mathrm{e}}^{2}-n_{\mathrm{o}}^{2}}{n_{\mathrm{e}}^{2}} \cos ^{2} \theta_{0} \sin ^{2} \phi_{0}\right)\left(\frac{k_{m}}{k}\right)^{2}}, \\
& c_{1}=\frac{\left[\left(k_{m} / k\right)^{2}-\varepsilon_{z z}\right] \varepsilon_{y x}+\left[\left(k_{m} / k\right)\left(k_{z 1} / k\right)+\varepsilon_{z x}\right] \varepsilon_{y z}}{\left[\left(k_{z 1} / k\right)^{2}+\left(k_{m} / k\right)^{2}-\varepsilon_{y y}\right]\left[\left(k_{m} / k\right)^{2}-\varepsilon_{z z}\right]-\varepsilon_{y z} \varepsilon_{z y}}, \\
& c_{2}=\frac{\left[\left(k_{m} / k\right)^{2}-\varepsilon_{z z}\right] \varepsilon_{x y}+\left[\left(k_{m} / k\right)\left(k_{z 2} / k\right)+\varepsilon_{x z}\right] \varepsilon_{z y}}{\left[\left(k_{z 2} / k\right)^{2}-\varepsilon_{x x}\right]\left[\left(k_{m} / k\right)^{2}-\varepsilon_{z z}\right]-\left[\left(k_{m} / k\right)\left(k_{z 2} / k\right)+\varepsilon_{z x}\right]\left[\left(k_{m} / k\right)\left(k_{z 2} / k\right)+\varepsilon_{x z}\right]} .
\end{aligned}
$$

Here, $m=1,2, d$ is the film thickness, $n_{\mathrm{o}}$ and $n_{\mathrm{e}}$ are the ordinary and extraordinary indexes, $\theta_{0}$ is the angle between the $x y$-plane and $\mathbf{c}_{0}$, and $\phi_{0}$ is the angle between the $x$-axis and the projection of $\mathbf{c}_{0}$ onto the $x y$-plane. The dielectric tensor of the medium can be written as

$$
\begin{gathered}
\varepsilon=\left[\begin{array}{lll}
\varepsilon_{x x} & \varepsilon_{x y} & \varepsilon_{x z} \\
\varepsilon_{y x} & \varepsilon_{y y} & \varepsilon_{y z} \\
\varepsilon_{z x} & \varepsilon_{z y} & \varepsilon_{z z}
\end{array}\right], \\
\varepsilon_{x x}=n_{\mathrm{o}}^{2}+\left(n_{\mathrm{e}}^{2}-n_{\mathrm{o}}^{2}\right) \cos ^{2} \theta_{0} \cos ^{2} \phi_{0}, \\
\varepsilon_{x y}=\varepsilon_{y x}=\left(n_{\mathrm{e}}^{2}-n_{\mathrm{o}}^{2}\right) \cos ^{2} \theta_{0} \sin \phi_{0} \cos \phi_{0}, \\
\varepsilon_{x z}=\varepsilon_{z x}=\left(n_{\mathrm{e}}^{2}-n_{\mathrm{o}}^{2}\right) \sin \theta_{0} \cos \theta_{0} \cos \phi_{0}, \\
\varepsilon_{y y}=n_{\mathrm{o}}^{2}+\left(n_{\mathrm{e}}^{2}-n_{\mathrm{o}}^{2}\right) \cos ^{2} \theta_{0} \sin ^{2} \phi_{0}, \\
\varepsilon_{y z}=\varepsilon_{z y}=\left(n_{\mathrm{e}}^{2}-n_{\mathrm{o}}^{2}\right) \sin \theta_{0} \cos \theta_{0} \sin \phi_{0},
\end{gathered}
$$




$$
\varepsilon_{z z}=n_{\mathrm{o}}^{2}+\left(n_{\mathrm{e}}^{2}-n_{\mathrm{o}}^{2}\right) \sin ^{2} \theta_{0}
$$

By setting

$$
\mathbf{E}(x, z) \equiv\left[\begin{array}{c}
A_{x} \exp \left(i \delta_{x}\right) \\
A_{y} \exp \left(i \delta_{y}\right)
\end{array}\right] \equiv\left[\begin{array}{c}
E_{x}^{\prime} \\
E_{y}^{\prime}
\end{array}\right],
$$

we obtain the polarization distribution in interference light with the use of the Stokes parameters as (Kliger, 1990)

$$
\left[\begin{array}{l}
S_{0} \\
S_{1} \\
S_{2} \\
S_{3}
\end{array}\right]=\left[\begin{array}{c}
A_{x}^{2}+A_{y}^{2} \\
A_{x}^{2}-A_{y}^{2} \\
2 A_{x} A_{y} \cos \left(\delta_{y}-\delta_{x}\right) \\
2 A_{x} A_{y} \sin \left(\delta_{y}-\delta_{x}\right)
\end{array}\right]
$$

Figure 2 illustrates the polarization distribution in the interference light calculated by varying $n_{\mathrm{o}}$ and $n_{\mathrm{e}}$. The paremeters used in this calculation are as follows: $d=10 \mu \mathrm{m}$, $\theta_{0}=0, \phi_{0}=\pi / 2, \lambda=532 \mathrm{~nm}, \theta_{1}=-\theta_{2}=1.5^{\circ} \quad$ (i.e., $\left.\left(\theta_{1}+\theta_{2}\right) / 2 \equiv \theta_{\mathrm{F}}=0\right), \mathbf{E}_{1}=(0,1)$ (i.e., $\mathbf{E}_{1}$ is s-polarized light), and $\mathbf{E}_{2}=(1,0)$ (i.e., $\mathbf{E}_{2}$ is $p$-polarized light). The one cycle of the interference pattern for the $x$-direction is given as $\Lambda_{x}=\lambda /\left|\sin \theta_{1}-\sin \theta_{2}\right| \cong 10 \mu \mathrm{m}$. As seen in Fig. 2, the polarization distribution of the interference light varies depending on values of $n_{\mathrm{o}}$ and $n_{\mathrm{e}}$. When $n_{\mathrm{e}}-n_{\mathrm{o}} \equiv \Delta n=0$, namely, the medium is isotorpic, the polarization state is modulated only for the $x$-direction. However, that is modulated for the $x$-and $z$-dirctions in the case of $\Delta n \neq 0$. This is because the refractive indexes for the $s$ - and $p$-polarized components differ in the anisotorpic medium since the optical axis is parallel to the incident plane. These calculated results clearly indicate that multidimensional periodic distribution of optical anisotorpy can be formed by the holographic recording. Therefore, we call these holograms, recorded in anisotropic media by vectrical light, 3D vector holograms.

Figure 3 illustrates interference patterns calculated by varing polarization states of the two beams in the case of $n_{\mathrm{o}}=1.52$ and $n_{\mathrm{e}}=1.75$. The other paremeters used in this calculation are the same as those mentioned above. In this chapter, linearly polarized (LP) light with the azimuth angle of $\pm 45^{\circ}$ for the $x$-axis are described as $q_{ \pm}$-polarized, respectively. In addition, right- and left-handed circularly polarized light are denoted by $r$ and $l$, respectively. The results shown in Fig. 3 demonstrate that various interference patterns are formed depending on polarization states of the two beams. When both the beams are spolarized, the intensity was modulated one-dimensionally and the polarization state was remained in the medium. In contrast, the polarization state was two-dimensionally modulated when both the beams are $q_{+}$- or $r$-polarized. This is because $s$-polarized light propagates with no change of polarization state since the electric field vector is parallel to the optical axis $\mathbf{c}_{0}$. Figure 4 shows interference patterns calculated by varying $\phi_{0}$ (i.e., the azimuth angle of $\mathbf{c}_{0}$ ). In this calculation, the two beams are $s$ - and $p$-polarized and the other parameters are the same as those mentioned above. As seen in Fig. 4, the interference 
pattern also depended on $\phi_{0}$. This fact suggests that the periodic structure, recorded by the $3 \mathrm{D}$ vector holography, is affected by the initial alignment of the medium.

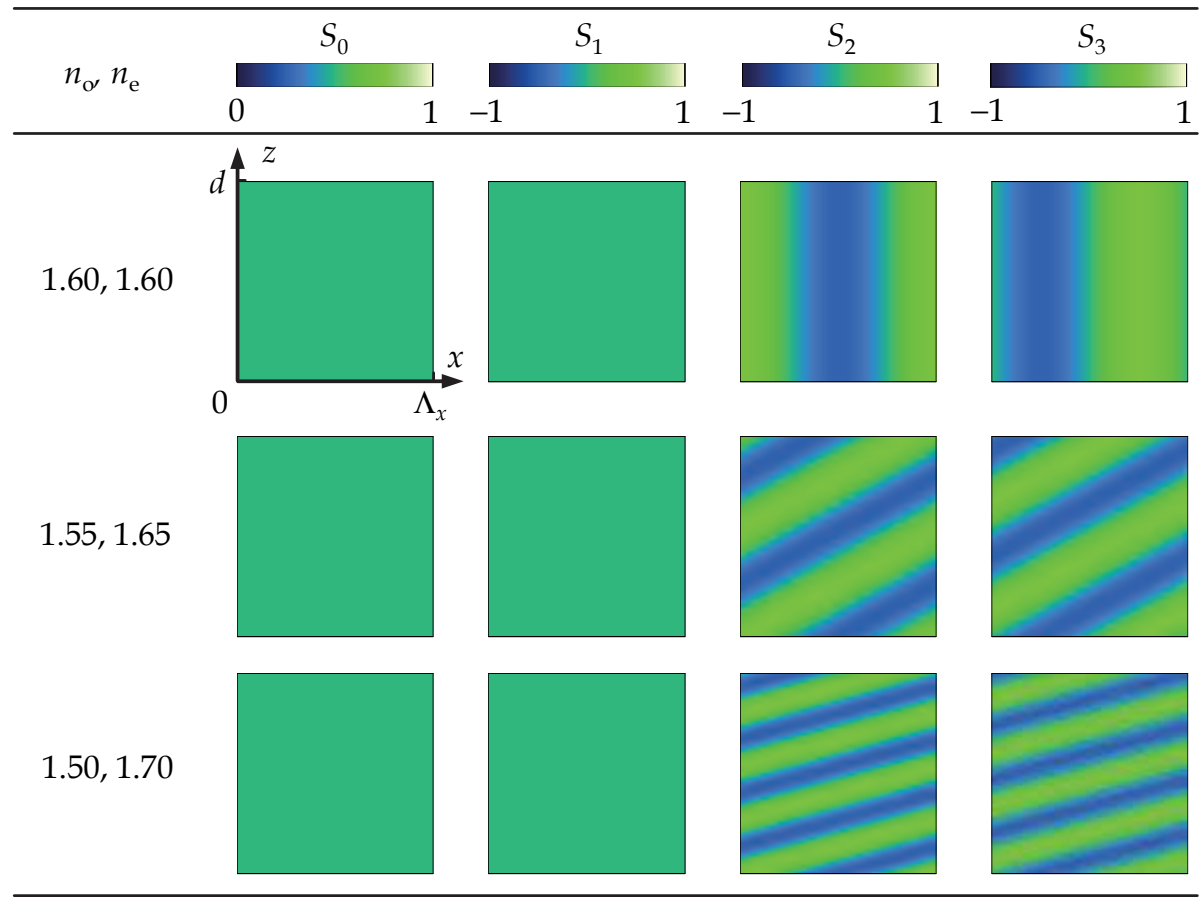

Fig. 2. Spatial distribution of the Stokes parameters in interference light calculated by varying the refractive indexes 


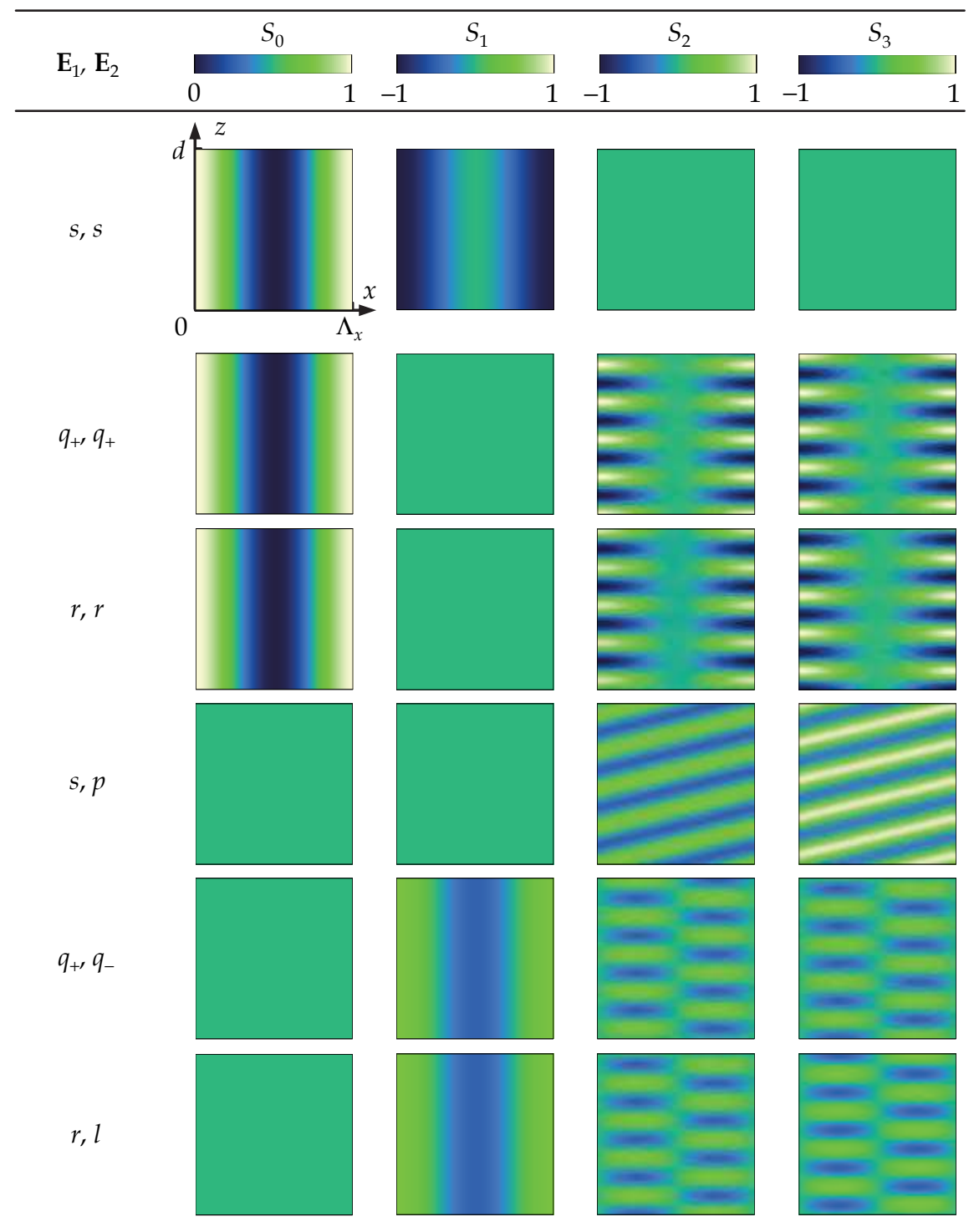

Fig. 3. Spatial distribution of the Stokes parameters in interference light calculated by varying polarization states of two recording beams 


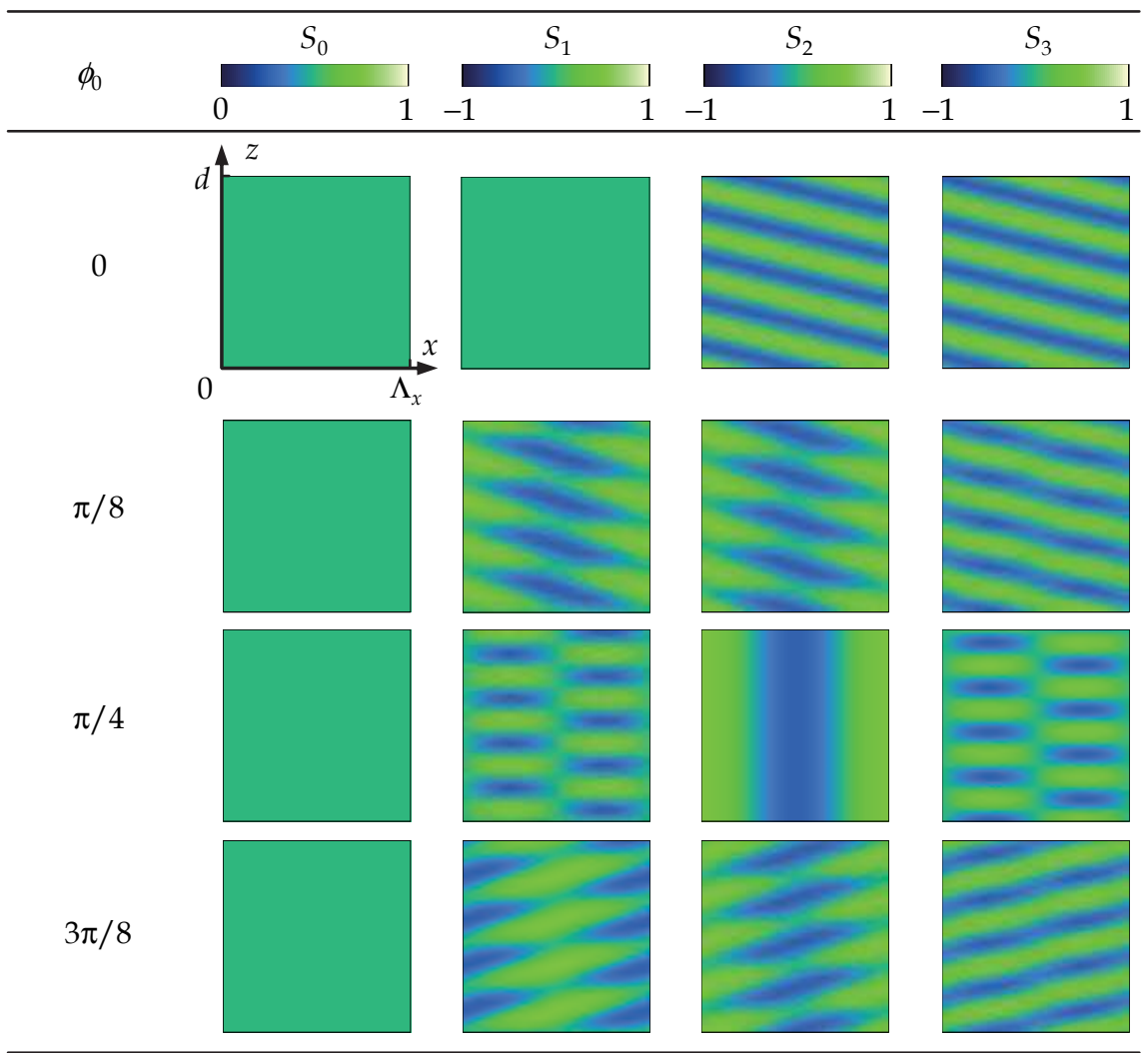

Fig. 4. Spatial distribution of the Stokes parameters in interference light calculated by varying the direction of the optical axis

In conclusion, we confirmed theoretically that $3 \mathrm{D}$ vector holography realizes fabrication of various multidimensional anisotropic structures owing to polarization states of recording beams, optical anisotorpy of the medium, and alignment of the optical axis.

\section{Photoreactive anisotropic medium with uniaxial anisotropy}

In order to confirm the principle described in section 2, a grating was recorded in a model medium by 3D vector holography and its diffraction properties were observed. We prepared an azo-dye doped liquid crystalline material as the photoreactive anisotropic medium by mixing nematic mixture E7, a side-chain liquid crystalline polymer (SLCP), and azo-dye 4-[N-(2-hydroxyethyl)-N-ethyl]amino-4'-nitroazobenzene, more commonly known as disperse red 1 (DR1), with a weight ratio of E7 : SLCP : DR1 $=59: 40: 1$. Here, E7 and DR1 were obtained from BDH-Merck and Ardrich, respectively. SLCP was synthesized using a poly(methyl methacrylate) backbone comprising 4-cyanophenyl benzoate side groups. The number and weight averages of SLCP used in this study were 11,700 and 32,800 , respectively. The chemical structures of the three components were illustrated in Fig. 5. They were stirred at around $100^{\circ} \mathrm{C}$ until a homogeneous solution was obtained. The 
mixture was sandwiched between two rubbed poly(vinyl alcohol)-coated glass substrates with $10 \mu \mathrm{m}$ thick spacers in order to obtain a homogeneously aligned sample. By using a polarizing optical microscope, we observed that the mixture exhibited the nematic phase at room temperature. Namely, it was confirmed that the azo-dye doped liquid crystalline material with homogeneous alignment was a uniaxial anisotropic medium.

(a)

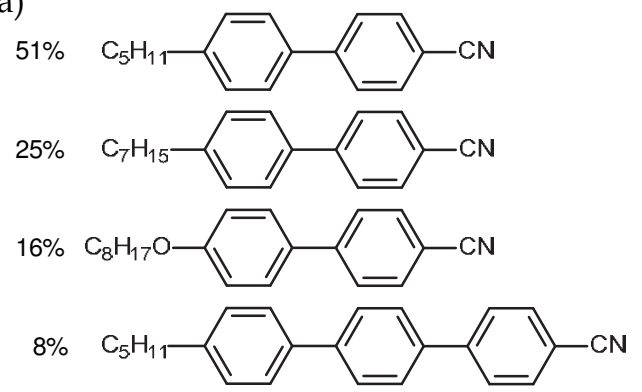

(b)

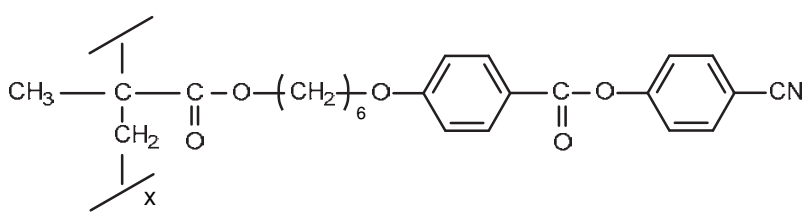

(c)

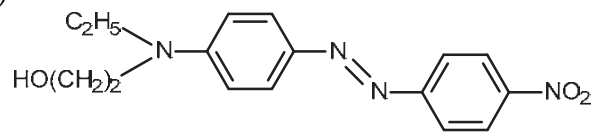

Fig. 5. Chemical structures of (a) E7, (b) SLCP, and (c) DR1

We consider the molecular reorientation induced by irradiation with polarized light. In order to discuss the polarization state of interference light, we assume that its propagation direction is in the $x z$-plane and that the angle between the $z$-axis and the propagation direction is given by

$$
\Theta_{\mathrm{F}}=\sin ^{-1}\left[\left(\sin \theta_{\mathrm{F}}\right) / \bar{n}\right],
$$

where $\bar{n}$ is the average refractive index of the medium. Here, we transform the coordinate system from $x y z$ into $x^{\prime} y^{\prime} z^{\prime}$ as follow

$$
\left[\begin{array}{l}
x^{\prime} \\
y^{\prime} \\
z^{\prime}
\end{array}\right]=\left[\begin{array}{ccc}
\cos \Theta_{\mathrm{F}} & 0 & \sin \Theta_{\mathrm{F}} \\
0 & 1 & 0 \\
-\sin \Theta_{\mathrm{F}} & 0 & \cos \Theta_{\mathrm{F}}
\end{array}\right]\left[\begin{array}{l}
x \\
y \\
z
\end{array}\right] \equiv \mathbf{R}^{-1}\left(\Theta_{\mathrm{F}}\right)\left[\begin{array}{l}
x \\
y \\
z
\end{array}\right] .
$$

In the $x^{\prime} y^{\prime} z^{\prime}$-coordinate system, the orientation direction (i.e., the optical axis) in the initial state is written as 


$$
\mathbf{c}_{0}^{\prime}=\mathbf{R}^{-1}\left(\Theta_{\mathrm{F}}\right) \mathbf{c}_{0},
$$

where $\mathbf{c}_{0}$ is the normalized vector of the initial optical axis in the $x y z$-coordinate system. Here, $\mathbf{c}_{0}^{\prime}$ can be described as

$$
\mathbf{c}_{0}^{\prime}=\left(\cos \theta_{0}^{\prime} \cos \phi_{0}^{\prime}, \cos \theta_{0}^{\prime} \sin \phi_{0}^{\prime}, \sin \theta_{0}^{\prime}\right),
$$

where $\theta_{0}^{\prime}$ is the angle between the $x^{\prime} y^{\prime}$-plane and $\mathbf{c}_{0}^{\prime}$ and $\phi_{0}^{\prime}$ is the angle between the $x^{\prime}$ axis and the projection of $\mathbf{c}_{0}^{\prime}$ onto the $x^{\prime} y^{\prime}$-plane. The reoriented optical axis in the $x^{\prime} y^{\prime} z^{\prime}$ coordinate system is written as

$$
\mathbf{c}_{\mathrm{re}}^{\prime}=\left(\cos \left(\theta_{0}^{\prime}+\theta_{\mathrm{re}}^{\prime}\right) \cos \left(\phi_{0}^{\prime}+\phi_{\mathrm{re}}^{\prime}\right), \cos \left(\theta_{0}^{\prime}+\theta_{\mathrm{re}}^{\prime}\right) \sin \left(\phi_{0}^{\prime}+\phi_{\mathrm{re}}^{\prime}\right), \sin \left(\theta_{0}^{\prime}+\theta_{\mathrm{re}}^{\prime}\right)\right),
$$

where $\theta_{\mathrm{re}}^{\prime}$ is the photoinduced tilt angle and $\phi_{\mathrm{re}}^{\prime}$ is the photoinduced azimuth angle. For simplicity, we assume that the amplitudes of the photoinduced angles are small and are proportional to the intensity of light. In addition, it has been revealed that the director of the azodye doped liquid crystalline material tilts to the perpendicular direction for the polarization azimuth. Therefore, using Equations 16 and 17, the two photoinduced angles are written as

$$
\begin{gathered}
\theta_{\mathrm{re}}^{\prime}=C_{\theta}\left|\mathbf{c}_{0}^{\prime} \cdot \mathbf{E}_{\mathrm{n}}\right| S_{0}, \\
\phi_{\mathrm{re}}^{\prime}=C_{\phi}\left|\mathbf{c}_{0}^{\prime} \cdot \mathbf{E}_{\mathrm{n}}\right|\left[S_{1} \sin \left(2 \phi_{0}^{\prime}\right)-S_{2} \cos \left(2 \phi_{0}^{\prime}\right)\right],
\end{gathered}
$$

where $C_{\theta}$ and $C_{\phi}$ are constants, which represent sensitivity to light intensity, and $\mathbf{E}_{\mathrm{n}}$ is the normalized electric field vector of interference light in the $x^{\prime} y^{\prime} z^{\prime}$-coordinate system, namely,

$$
\mathbf{E}_{\mathrm{n}}=\frac{1}{\sqrt{\left|E_{x}^{\prime}\right|^{2}+\left|E_{y}^{\prime}\right|^{2}}}\left[\begin{array}{c}
E_{x}^{\prime} \\
E_{y}^{\prime} \\
0
\end{array}\right] \text {. }
$$

In the $x y z$-coordinate system, the reoriented optical axis is given by

$$
\mathbf{c}_{\mathrm{re}}=\mathbf{R}\left(\Theta_{\mathrm{F}}\right) \mathbf{c}_{\mathrm{re}}^{\prime} \equiv\left(c_{x}, c_{y}, c_{z}\right)=(\cos \theta \cos \phi, \cos \theta \sin \phi, \sin \theta),
$$

where $\theta$ is the angle between the $x y$-plane and $\mathbf{c}_{\mathrm{re}}$ and $\phi$ is the angle between the $x$-axis and the projection of $\mathbf{c}_{\mathrm{re}}$ onto the $x y$-plane. From Equation 26, we obtain

$$
\begin{gathered}
\theta(x, z)=\sin ^{-1} c_{z}, \\
\phi(x, z)=\sin ^{-1}\left(c_{y} / \cos \theta\right),
\end{gathered}
$$

Therefore, the photoinduced tilt and azimuth angles in the xyz-coordinate system are obtained as

$$
\theta_{\text {re }}(x, z)=\theta-\theta_{0}
$$




$$
\phi_{\text {re }}(x, z)=\phi-\phi_{0}
$$

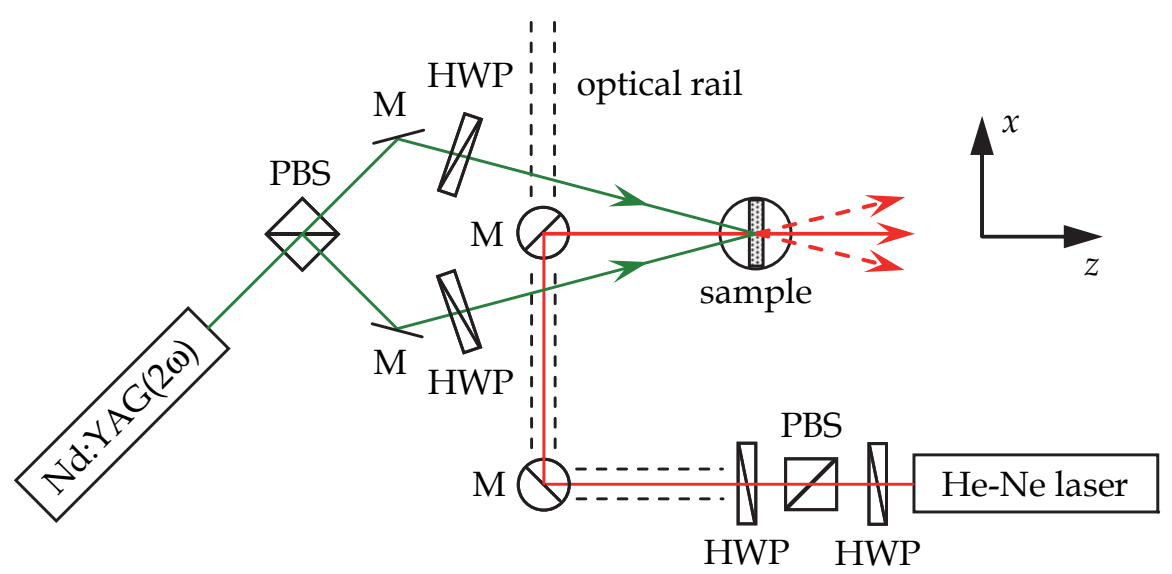

Fig. 6. Schematic illustration of the experimental setup (HWP, half-wave plate; PBS, polarizing beam splitter; $\mathrm{M}$, mirror). Circles represent rotary stage in the incident plane

\section{Observed diffraction properties}

Figure 6 schematically illustrates the experimental setup for the holographic recording and the reconstruction. A frequency doubled, Nd-doped yttrium aluminum garnet laser with an operating wavelength of $532 \mathrm{~nm}$ was used for recording the hologram. This is because the azo-dye effectively reacts to irradiation with green light. The LP recording beam was divided into $s$ - and $p$ - polarizations using a polarizing beam splitter, and the two beams intersected on the sample as illustrated in Fig. 6. The intensity of the two recording beams was $900 \mathrm{~mW} / \mathrm{cm}^{2}$, respectively. The reconstruction properties were probed by an LP He-Ne laser with a wavelength of $633 \mathrm{~nm}$. The intensity of diffracted beams was measured by a photodiode detector while varying the incident angle of the probe beam. All measurements were conducted at room temperature.

Figure 7 presents the measured positive and negative first-order diffraction efficiencies for $\theta_{1}=-\theta_{2}=1.5^{\circ}$ (i.e., $\left.\theta_{\mathrm{F}}=0\right), \theta_{0}=0$, and $\phi_{0}=\pi / 2$. The \pm 1 st-order diffraction efficiencies $\eta_{ \pm}$ were defined as the ratio of the intensity of the diffracted beam to the total intensity of the transmitted light. As seen in Fig. 7, strong diffraction appeared in the \pm 1 st-order when the probe beam was $p$ - and s-polarized, respectively. When the probe beams was $q_{+}$-polarized, relatively strong diffraction was observed in both the orders. The results shown in Fig. 7 clearly indicate that the diffraction efficiency depends on the incident angle of the probe beam $\theta_{\text {in }}$. It is also important to remember that the angular dependence is asymmetry for $\theta_{\text {in }}=0$ even though $\theta_{\mathrm{F}}=0$. Figure 8 illustrates observed polarization states of the diffracted beams in the case of $\theta_{\text {in }}=0$. This result implies that the grating diffracts $p$ - and s-polarized components of incident light as $s$ - and $p$-polarized light, respectively. Here, the $p$ - and s-polarizations were defined for the $x z$-plane in this chapter. This property with polarization discrimination is useful for applying to optical elements such as a polarizing beam splitter with a thin thickness. 


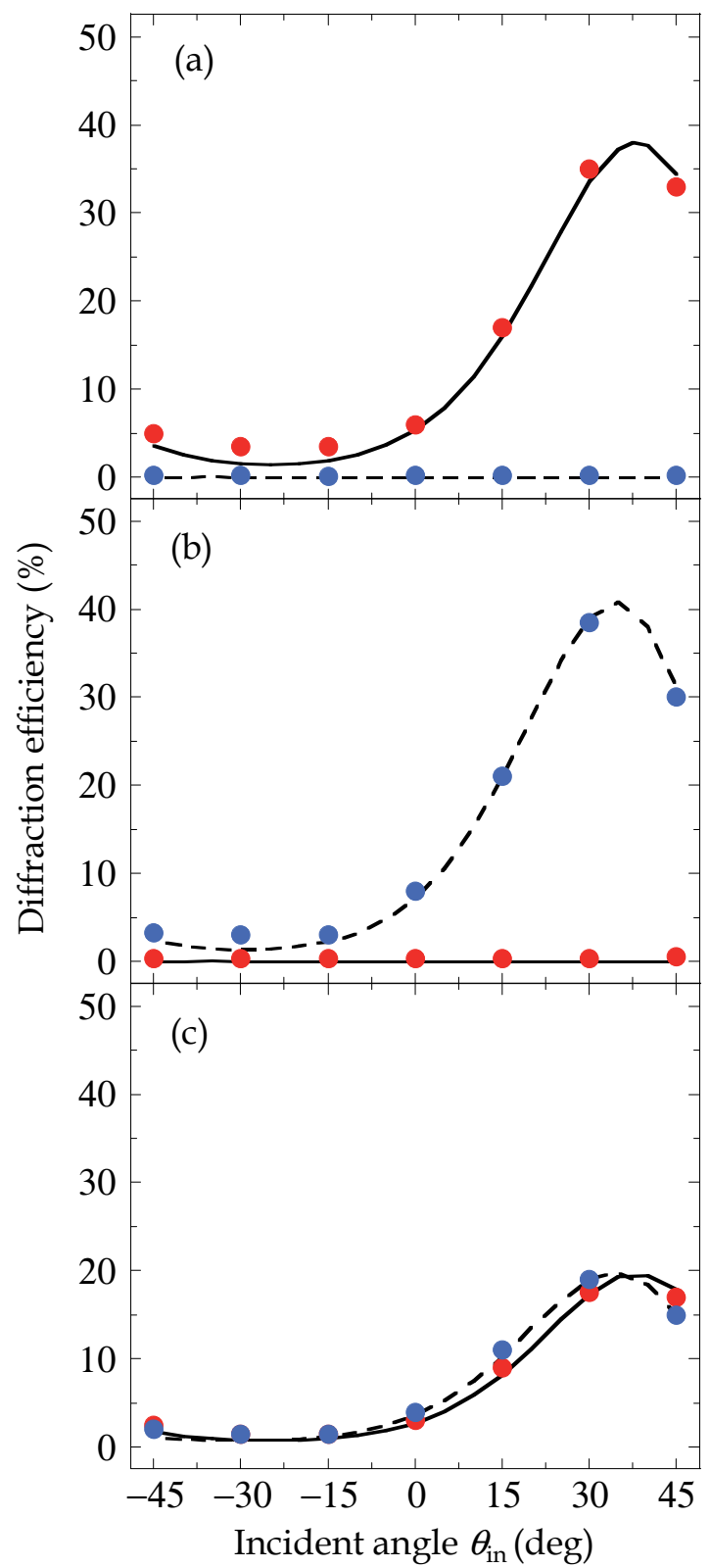

Fig. 7. Angular dispersion of the diffraction efficiency for (a) $p_{-}$, (b) $s^{-}$, and (c) $q_{+}$-polarized probe beam. Red and blue plots represent the measured positive and negative first-order diffraction efficiencies. Solid and dashed lines represent the calculated positive and negative first-order diffraction efficiencies 
(a)

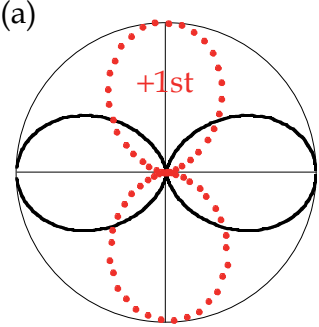

(b)

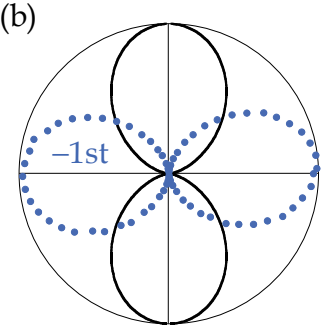

(c)

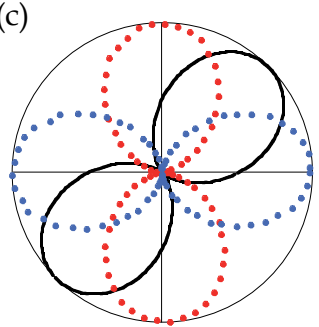

Fig. 8. Polar plots of polarization states for incident and diffraction beams. The incident beam, indicated as solid lines, is (a) $p$-, (b) $s_{-}$, and (c) $q_{+}$-polarized. Red and blue plots represent measured data for positive and negative first-order diffraction beams

\section{Theoretical characterization}

\subsection{FDTD method}

In order to characterize the observed results mentioned above, optical behaviour of the periodical distribution of the dielectric tensor, which was described in section 3, were calculated by employing the FDTD method. This method imposes no limitations on the dimension of the object and the incident conditions since Maxwell's equations are directly solved numerically by the calculus of finite differences in the algorithm (Yee, 1966; Teflova \& Gedne, 2005). The FDTD method can analyze anisotropic media such as liquid crystals (Kriezis \& Elston, 1999, 2000; Titus et al., 2001; Scharf, 2007). In addition, it has been reported that the FDTD method is suitable for characterizing anisotropic gratings recorded by vector holography (Oh \& Escuti, 2006, 2007; Ono et al. 2008a, 2008b).

Figure 9 schematically illustrates the model for the FDTD calculation in this study. We set the calculation conditions as follows: the wavelength of the incident light is $633 \mathrm{~nm}$, the size of the analytical area ( $x$-direction $\times z$-direction) is $162 \mu \mathrm{m} \times 15 \mu \mathrm{m}$, the grid spacing is 20 $\mathrm{nm} \times 20 \mathrm{~nm}$, and the time step is $0.047 \mathrm{fs}$. As the boundary condition, the perfectly matched layer (PML) with the thickness of $1 \mu \mathrm{m}$ was used (Teflova \& Gedne, 2005). The glass substrates and the alignment layers were ignored and antireflection (AR) layers were installed at the air-hologram boundaries for reducing the effect of multiple interference (Southwell, 1983). The diffraction properties in the far field were calculated by Fourier transforming each component of the electric field at the output plane.

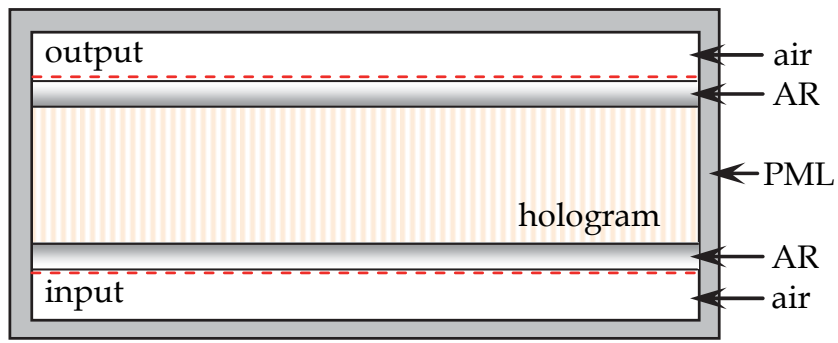

Fig. 9. Schematic layout of the analytical area for the FDTD calculation (AR, anti-reflection coatings; PML, perfectly matched layer) 


\subsection{Analysis of observed diffraction properties}

The calculated diffraction efficiencies for the experimental conditions, described in section 4, were shown in Fig. 7. The parameters used this calculation are as follows: $n_{\mathrm{o}}=1.52$, $n_{\mathrm{e}}=1.75, C_{\theta}=4.9 \times 10^{-2} \mathrm{~cm}^{2} / \mathrm{W}$, and $C_{\phi}=4.8 \times 10^{-2} \mathrm{~cm}^{2} / \mathrm{W}$. As seen in Fig. 7 , the observed diffraction efficiencies were well explained with the use of the theoretical model and the FDTD calculation. This fact suggests that the $3 \mathrm{D}$ vector hologram is actually recorded in the azo-dye doped liquid crystalline material. The calculated distribution of the photoinduced tilt and azimuth angles were illustrated in Fig. 10. It is clearly known that a slanted grating is formed based on the spatially varying state of polarization. Figure 11 illustrates the calculated distribution of electric fields in and around the hologram when the incident beam is $p$ - and s-polarized. Here, the AR layers were not implemented in this calculation to accentuate the air-hologram boundaries. For the $p$-polarization, the s-component was gradually generated in the hologram and propagated to the positive first-order direction. In contrast, the $p$-component was gradually generated in the hologram and propagated to the negative first-order direction when the incident light was s-polarized. The calculated results are consistent with the observed property of polarization conversion shown in Fig. 8. This fact also demonstrates the accuracy of the theoretical model.

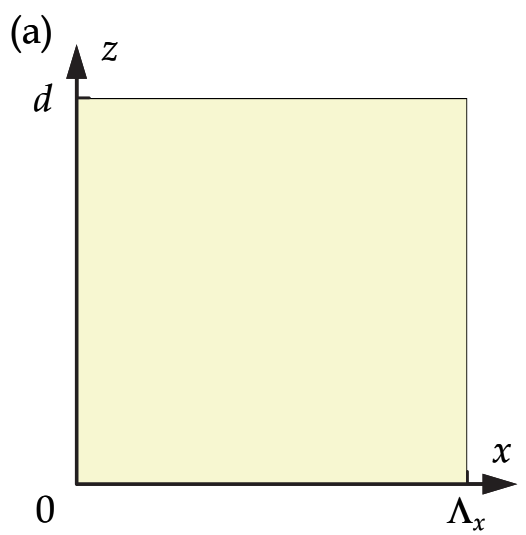

(b)

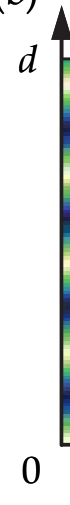

(c)

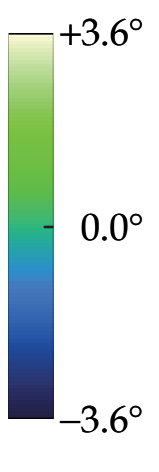

Fig. 10. Calculated distribution of (a) $\theta_{\mathrm{re}}$ and (b) $\phi_{\mathrm{re}}$ and (c) their value 


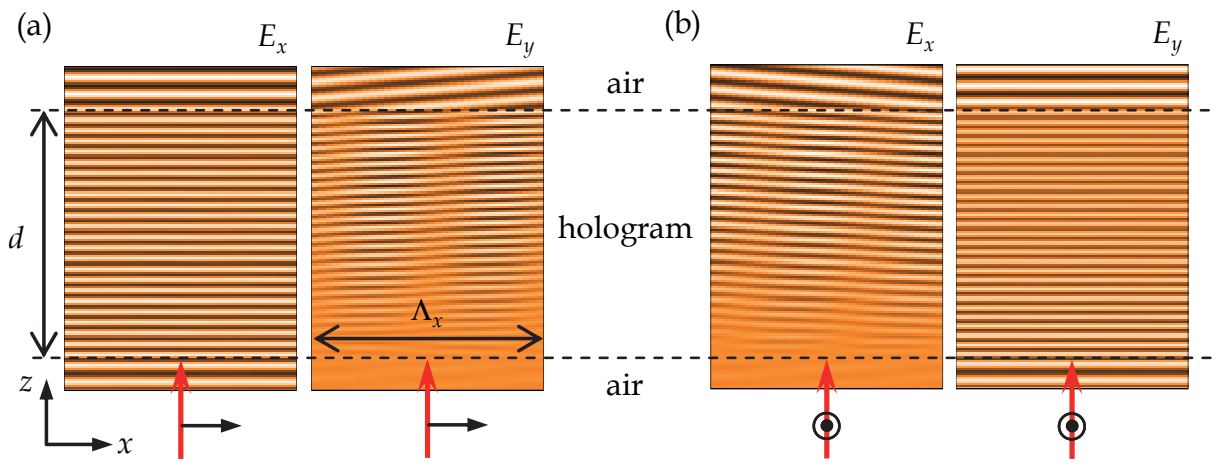

Fig. 11. Calculated electric fields for the (a) $p$ - and (b) s-polarized probe beam. $E_{x}$ and $E_{y}$ are the real part of the $x$-and $y$-components for the electric field vector

\subsection{Simulated diffraction properties}

This section investigates the relationship between various parameters of the $3 \mathrm{D}$ vector hologram and its diffraction properties through the theoretical model. Figure 12 presents the calculated results of the grating structure for varying $\Lambda_{x}$. The grating pitch in the grating $\Lambda$ and the slanted angle $\Theta_{S}$, which is the angle between the $x$-axis and the grating vector, are given by

$$
\Lambda=\Lambda_{z} \cos \Theta_{\mathrm{S}}=\Lambda_{z} \cos \left(\tan ^{-1} \frac{\Lambda_{z}}{\Lambda_{x}}\right),
$$

where $\Lambda_{z}$ is the grating pitch for the $z$-axis. In this case, $\Lambda$ and $\Lambda_{z}$ are determined by the distribution of $S_{2}$ and $S_{3}$ as described in Equations 23 and 24. We confirmed that $\Lambda$ and $\Lambda_{z}$ decreased with decreasing $\Lambda_{x}$. It should also be pointed out that $\Lambda_{z} \cong \Lambda_{x} / \Delta n=2.31 \mu \mathrm{m}$ for relatively large $\Lambda_{x}$ (namely, for $\left|\theta_{1}\right|=\left|\theta_{2}\right|<<1$ ).
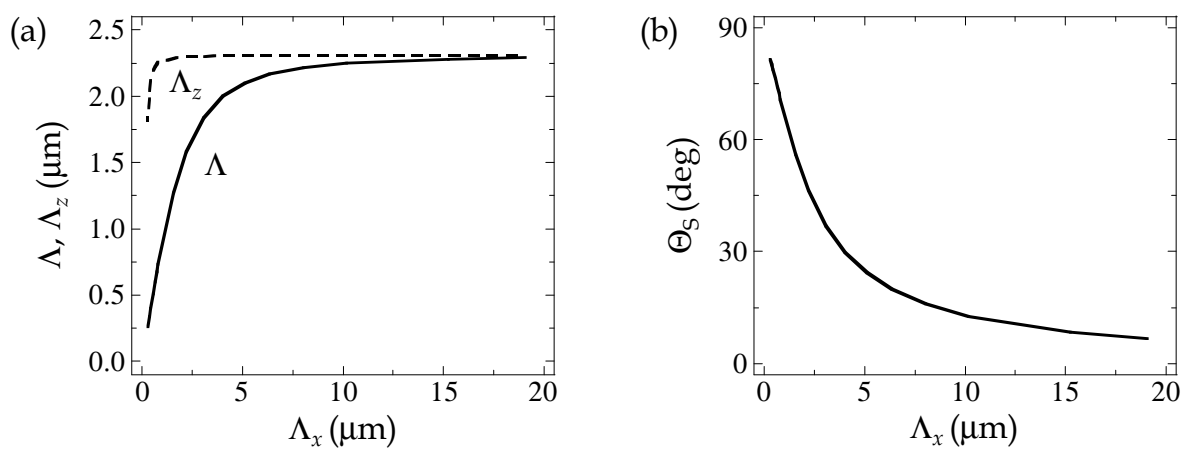

Fig. 12. Dependence of (a) $\Lambda, \Lambda_{z}$, and (b) $\Theta_{S}$ on $\Lambda_{x}$

Figure 13 illustrates angular dispersion of $\eta_{ \pm 1}$ calculated for $\Lambda_{x}=2,4,8 \mu \mathrm{m}$ when the probe beam is $p$ - and $s$-polarized. The maximum values of the diffraction efficiency 
$\eta_{ \pm 1}\left(\theta_{\text {in }}\right) \equiv \eta_{ \pm 1}\left(\theta_{\max }\right)$ were almost the same for respective $\Lambda_{x}$. However, $\theta_{\max }$ varied by $\Lambda_{x}$ as seen in Fig. 13. In addition, $\theta_{\max }$ was remarkably influenced by the polarization state of the probe beam. For example, $\theta_{\max }$ at $\Lambda_{x}=2 \mu \mathrm{m}$ were approximately $21^{\circ}$ and $2^{\circ}$ when the probe beam was $p$ - and s-polarized. We attribute the reason for the discrepancy to a change of the Bragg angle owing to the difference of the refractive index for the two polarizations. This is one of a characteristic of the $3 \mathrm{D}$ vector hologram.

(a)

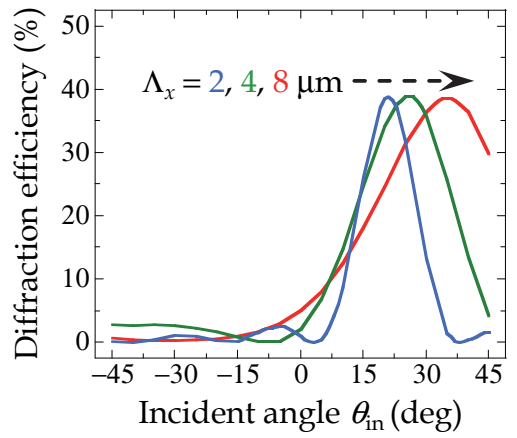

(b)

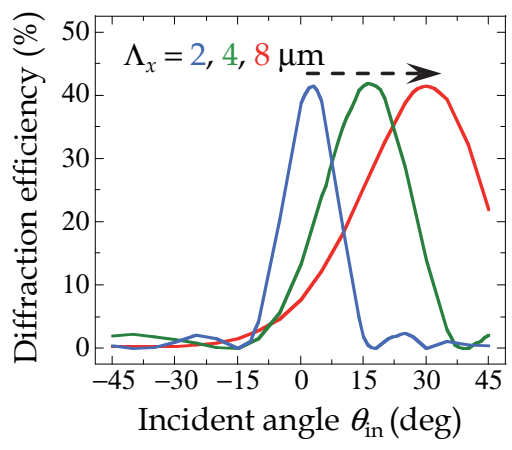

Fig. 13. Angular dependence of the diffraction efficiency calculated by varying $\Lambda_{x}$. (a) $\eta_{+}$ for $p$-polarization. (b) $\eta_{-}$for s-polarization

Figure 14 presents $\eta_{+1}\left(\theta_{\text {in }}\right)$ for the $p$-polarized probe beam calculated by varying the film thickness $d$. It is clearly shown in Fig. 14 that the angular dependence of the diffraction efficiency decreases with increasing $\Lambda_{x}$ (i.e., $\Lambda$ ) and decreasing $d$. This is understood by considering the difference of thick and thin gratings, similarly common diffraction gratings in isotropic media (Kogelnik, 1969; Hariharan, 1996). Figure 15 illustrates dependence of $\eta_{+1}$ at $\theta_{\text {in }}=\theta_{\max }=24.7^{\circ}$ and $\eta_{-1}$ at $\theta_{\text {in }}=\theta_{\max }=-12.5^{\circ}$ on $d$ in the case of $\Lambda_{x}=1 \mu \mathrm{m}$. As seen in Fig. 15, $\eta_{+1}$ for the $p$-polarization and $\eta_{-1}$ for the s-polarization reached about $100 \%$ around $d=22 \mu \mathrm{m}$ and then decreased with increasing $d$, respectively. These curves were well fitted sinusoidal functions similarly transmission type thick gratings in anisotropic media. In contrast, it was confirmed that $\eta_{+1}$ for the s-polarization and $\eta_{-1}$ for the $p$-polarization were nearly equal to zero, respectively.

(a)

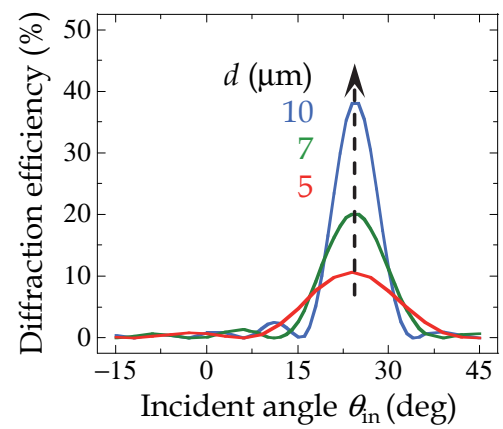

(b)

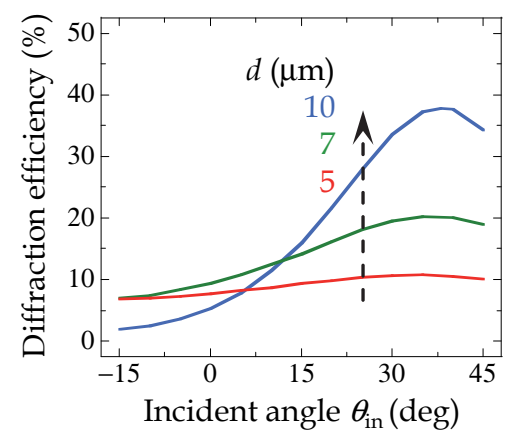

Fig. 14. Angular dependence of $\eta_{+}$for $p$-polarization at (a) $\Lambda_{x}=1 \mu \mathrm{m}$ and (b) $\Lambda_{x}=10 \mu \mathrm{m}$ 
(a)

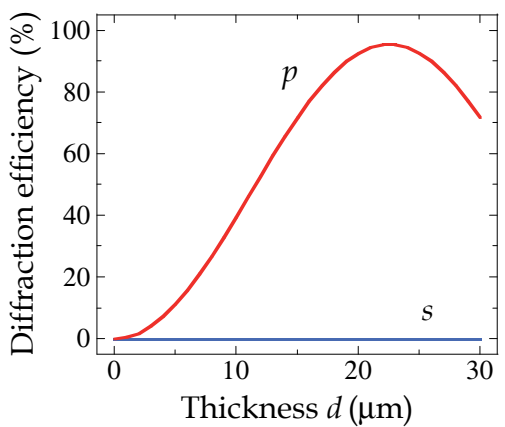

(b)

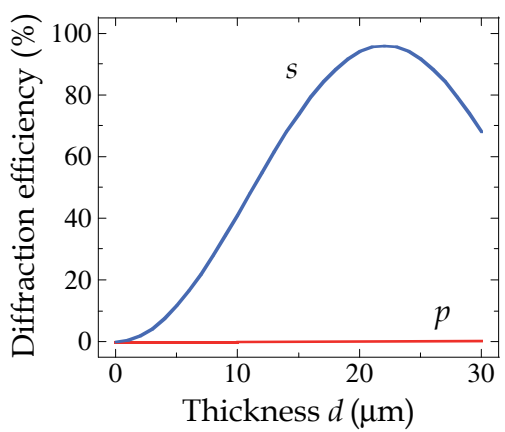

Fig. 15. Dependence of (a) $\eta_{+}$at $\theta_{\text {in }}=24.7^{\circ}$ and (b) $\eta_{-}$at $\theta_{\text {in }}=-12.5^{\circ}$ on the film thickness. Red and blue lines are the calculated results for $p$ - and s-polarizations

\section{Conclusions}

In this chapter, the principle of 3D vector holography has been described. We considered interference of two polarized plane waves with coherency in anisotropic media, and formulated a type of 3D vector hologram as spatial distribution of the dielectric tensor based on the polarization state of interference light. Optical behaviour of the periodic distribution was simulated by employing the FDTD method and the result was indeed consistent with the observed diffraction properties of the holographic grating, recorded in an azo-dye doped liquid crystalline medium with uniaxial optical anisotropy. Hence, we confirmed that various periodic structures, consisted of spatial distribution of optical anisotropy, could be formed by means of the $3 \mathrm{D}$ vector holography. Furthermore, detailed diffraction properties of the holographic grating were studied with the use of the FDTD method. As a result, it was revealed that the 3D vector hologram exhibit various useful characteristics including high diffraction efficiencies, angular dispersion, polarization dependence, and polarization conversion. We believe that the $3 \mathrm{D}$ vector holography realizes fabrication of higher-order structures of optical anisotropy and that these structures can be applied to diffractive optical elements with multiple functions, 3D optical memories, anisotropic photonic crystals and so on.

\section{References}

Asatryan, K. E., Frédérick, S., Galstian, T., \& Vallée, R. (2004). Recording of polarization holograms in photodarkened amorphous chalcogenide films. Appl. Phys. Lett., Vol. 84, No. 10, (March 2004), pp. 1626-1628, ISSN 0003-6951

Birabassov, R. \& Galstian, T. V. (2001). Analysis of the recording and reconstruction of the polarization state of light. J. Opt. Soc. Am. B, Vol. 18, No. 10, (October 2001), pp. 1423-1427, ISSN 0740-3224

Ebralidze, T. D. \& Ebralidze, N. A. (1992). Hologram record by means of film anisotropy photoinduction. Appl. Opt., Vol. 31, No. 23, (August 1992), pp. 4720-4724, ISSN 0003-6935

Hariharan, P. (1996). Optical holography: principles, techniques, and applications. Cambridge Univ. Press, ISBN 0-521-43348-7, New York 
Huang, T. \& Wagner, K. H. (1993). Holographic diffraction in photoanisotropic organic materials. J. Opt. Soc. Am. A, Vol. 10, No. 2, (February 1993), pp. 306-315, ISSN 07403232

Kim, D. Y., Tripathy, S. K., Li, L., \& Kumar, J. (1995). Laser-induced holographic surface relief gratings on nonlinear optical polymer films, Appl. Phys. Lett., Vol. 66, No. 10, (March 1995), pp. 1166-1168, ISSN 0003-6951

Kliger, D. S., Lewis, J. W., \& Randall, C. E. (1990). Polarized light in optics and spectroscopy. Academic press, ISBN 0-12-414975-8, California

Kogelnik, H. (1969). Coupled wave theory for thick hologram gratings. Bell Syst. Tech. J., Vol. 48, No. 8, pp. 2909-2947, ISSN 0005-8580

Kriezis, E. E. \& Elston, S. J. (1999). Finite-difference time domain method for light wave propagation within liquid crystal devices. Opt. Commun., Vol. 165, No. 1-3, (July 1999), pp. 99-105, ISSN 0030-4018

Kriezis, E. E. \& Elston, S. J. (2000). Light wave propagation in liquid crystal displays by the 2-D finite-difference time-domain method. Opt. Commun., Vol. 177, No. 1-6, (April 2000), pp. 69-77, ISSN 0030-4018

Labarthet, F. L., Buffeteau, T., \& Sourisseau, C. (1998). Analyses of the diffraction efficiencies, birefringence, and surface relief gratings on azobenzene-containing polymer films. J. Phys. Chem. B, Vol. 102, No. 15, (April 1998), pp. 2654-2662, ISSN 1089-5647

Labarthet, F. L., Buffeteau, T., \& Sourisseau, C. (1999). Azopolymer holographic diffraction gratings: time dependent analyses of the diffraction efficiency, birefringence, and surface modulation induced two linearly polarized interfering beams. J. Phys. Chem. B, Vol. 103, No. 32, (August 1999), pp. 6690-6699, ISSN 1089-5647

Lien, A. (1990). Extended Jones matrix representation for the twisted nematic liquid-crystal display at oblique incidence. Appl. Phys. Lett., Vol. 57, No. 26, (December 1990), pp. 2767-2769, ISSN 0003-6951

Lien, A. (1997). A detailed derivation of extended Jones matrix representation for twisted nematic liquid crystal displays. Liq. Cryst., Vol. 22, No. 2, (February 1997), pp. 171175, ISSN 0267-8292

Naydenova, I., Nikolova, L., Todorov, T. Holme, N. C. R., Ramanujam, P. S., \& Hvilsted, S. (1998). Diffraction from polarization holographic gratings with surface relief in side-chain azobenzene polyesters, J. Opt. Soc. Am. B, Vol. 15, No. 4, (April 1998), pp. 1257-1265, ISSN 0740-3224

Nikolova, L. \& Todorov, T. (1984). Diffraction efficiency and selectivity of polarization holographic recording. Opt. Acta, Vol. 31, No. 5, (May 1984), pp. 579-588, ISSN 0030-3909

Nikolova, L., Todorov, M., Ivanov, M., Andruzzi, F., Hvilsted, S., \& Ramanujam, P. S. (1996). Polarization holographic gratings in side-chain azobenzene polyesters with linear and circular photoanisotropy. Appl. Opt., Vol. 35, No. 20, (July 1996), pp. 3835-3840, ISSN 0003-6935

Nikolova, L., Todorov, M., Ivanov, M., Andruzzi, F., Hvilsted, S., \& Ramanujam, P. S. (1997). Photoinduced circular anisotropy in side-chain azobenzene polyesters. Opt. Matter., Vol. 8, No. 4, (November 1997), pp. 255-258, ISSN 0925-3467

Oh, C. \& Escuti, M. J. (2006). Time-domain analysis of periodic anisotropic media at oblique incidence: an efficient FDTD implementation. Opt. Express, Vol. 14, No. 24, (November 2006), pp. 11870-11884, ISSN 1094-4087 
Oh, C. \& Escuti, M. J. (2007). Numerical analysis of polarization gratings using the finitedifference time-domain method. Phys. Rev. A, Vol. 76, No. 043815, (October 2007), pp. 1-8, ISSN 1050-2947

Ono, H., Sekiguchi, T., Emoto, A., \& Kawatsuki, N. (2008). Light wave propagation in polarization holograms formed in photoreactive polymer liquid crystals. Jpn. J. Appl. Phys., Vol. 47, No. 5, (May 2008), pp. 3559-3563, ISSN 0021-4922

Ono, H., Sekiguchi, T., Emoto, A., Shioda, T., \& Kawatsuki, N. (2008). Light wave propagation and Bragg diffraction in thick polarization gratings. Jpn. J. Appl. Phys., Vol. 47, No. 10, (October 2008), pp. 7963-7967, ISSN 0021-4922

Ono, H., Wakabayashi, H., Sasaki, T., Emoto, A., Shioda, T., \& Kawatsuki, N. (2009). Vector holograms using radially polarized light. Appl. Phys. Lett., Vol. 94, No. 071114, (February 2009), pp. 1-3, ISSN 0003-6951

Ono, H., Suzuki, K., Sasaki, T., Iwato, T., Emoto, A., Shioda, T., \& Kawatsuki, N. (2009). Reconstruction of polarized optical images in two- and three-dimensional vector holograms. J. Appl. Phys., Vol. 106, No. 083109, (October 2009,) pp. 1-7, ISSN 00218979

Provenzano, C., Cipparrone, G., \& Mazzulla, A. (2006). Photopolarimeter based on two gratings recorded in thin organic films. Appl. Opt., Vol. 45, No. 17, (June 2006), pp. 3929-3933, ISSN 0003-6935

Ramanujam, P. S., Holme, N. C. R., \& Hvilsted, S. (1996). Atomic force and optical near-field microscopic investigations of polarization holographic gratings in a liquid crystalline azobenzene side-chain polyester, Appl. Phys. Lett., Vol. 68, No. 10, (March 1996), pp. 1329-1331, ISSN 0003-6951

Rochon, P., Batalla, E., \& Natansohn, A. (1995). Optically induced surface gratings on azoaromatic polymer films, Appl. Phys. Lett., Vol. 66, No. 2, (January 1995), pp. 136138, ISSN 0003-6951

Samui, A. B. (2008). Holographic recording medium. Recent Patents on Mater. Sci., Vol. 1, No. 3, pp. 79-94, ISSN 1874-4648.

Sasaki, T., Ono, H., \& Kawatsuki, N. (2008). Three-dimensional vector holograms in anisotropic photoreactive liquid-crystal composites. Appl. Opt., Vol. 47, No. 13, (April 2008), pp. 2192-2200, ISSN 0003-6935

Scharf, T. (2007). Polarized light in liquid crystals and polymers. Wiley, ISBN 978-0-471-74064-3, New Jersey

Taflove, A. \& Gedne, S. C. (2005). Computational electrodynamics: the finite-difference timedomain method. Artech House, ISBN 1-58053-832-0, Massachusetts

Titus, C. M., Kelly, R., Gartland, E. C., Shiyanovskii, S. V., Anderson, J. A., \& Bos, P. J. (2001). Asymmetric transmissive behaviour of liquid-crystal diffraction gratings. Opt. Lett., Vol. 26, No. 15, (August 2001), pp. 1188-1190, ISSN 0146-9592

Todorov, T., Nikolova, L., \& Tomova, N. (1984). Polarization holography. 2: Polarization holographic gratings in photoanisotropic materials with and without intrinsic birefringence. Appl. Opt., Vol. 23, No. 24, (December 1984), pp. 4588-4591, ISSN 0003-6935

Yee, K. S. (1966). Numerical solution of initial boundary value problems involving Maxwell's equations in isotropic media. IEEE Trans. Antennas Propag., Vol. 14, No. 3, pp. 302-307, ISSN 0018-926X 


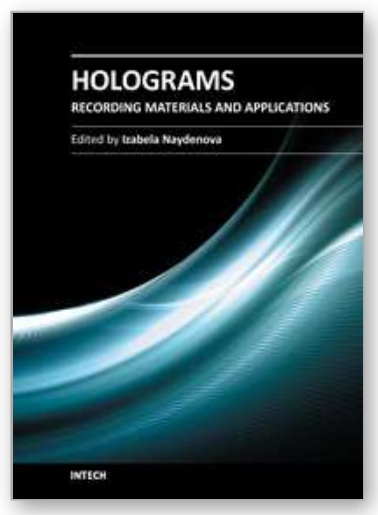

\author{
Holograms - Recording Materials and Applications \\ Edited by Dr Izabela Naydenova
}

ISBN 978-953-307-981-3

Hard cover, 382 pages

Publisher InTech

Published online 09, November, 2011

Published in print edition November, 2011

Holograms - Recording Materials and Applications covers recent advances in the development of a broad range of holographic recording materials including ionic liquids in photopolymerisable materials, azo-dye containing materials, porous glass and polymer composites, amorphous chalcogenide films, Norland optical adhesive as holographic recording material and organic photochromic materials. In depth analysis of collinear holographic data storage and polychromatic reconstruction for volume holographic memory are included. Novel holographic devices, as well as application of holograms in security and signal processing are covered. Each chapter provides a comprehensive introduction to a specific topic, with a survey of developments to date.

\title{
How to reference
}

In order to correctly reference this scholarly work, feel free to copy and paste the following:

Tomoyuki Sasaki, Akira Emoto, Kenta Miura, Osamu Hanaizumi, Nobuhiro Kawatsuki and Hiroshi Ono (2011). Three-Dimensional Vector Holograms in Photoreactive Anisotropic Media, Holograms - Recording Materials and Applications, Dr Izabela Naydenova (Ed.), ISBN: 978-953-307-981-3, InTech, Available from: http://www.intechopen.com/books/holograms-recording-materials-and-applications/three-dimensional-vectorholograms-in-photoreactive-anisotropic-media

\section{INTECH}

open science | open minds

\section{InTech Europe}

University Campus STeP Ri

Slavka Krautzeka 83/A

51000 Rijeka, Croatia

Phone: +385 (51) 770447

Fax: +385 (51) 686166

www.intechopen.com

\section{InTech China}

Unit 405, Office Block, Hotel Equatorial Shanghai

No.65, Yan An Road (West), Shanghai, 200040, China 中国上海市延安西路65号上海国际贵都大饭店办公楼 405 单元

Phone: +86-21-62489820

Fax: $+86-21-62489821$ 
(C) 2011 The Author(s). Licensee IntechOpen. This is an open access article distributed under the terms of the Creative Commons Attribution 3.0 License, which permits unrestricted use, distribution, and reproduction in any medium, provided the original work is properly cited. 